\title{
Recorridos chamánicos: sobre el afecto cognitivo en Arguedas, W. H. Hudson, y Deleuze y Guattari
}

\author{
Shamanic Journeys: on Cognitive Affection in Arguedas,
} W.H. Hudson, and Deleuze and Guattari

Decursos xamanistas: sobre o afeto cognitivo em Arguedas, W. H. Hudson, e Deleuze e Guattari

\section{Sara Castro-Klarén}

JOHNS HOPKINS UNIVERSITY

Profesora de Literatura y Cultura Latinoamericana en Johns Hopkins

University. PhD en Literatura por la Universidad de California, Los

Ángeles. Sus publicaciones incluyen El mundo mágico de fosé María

Arguedas (Instituto de Estudios Peruanos, 1973), Understanding Mario

Vargas Llosa (University of South Carolina Press, 1990), Escritura, sujeto y transgresión en la literatura latinoamericana (Premià, 1989). Su libro más reciente es The Narrow Pass of Our Nerves: Writing, Coloniality and Postcolonial Theory (Vervuert, 2011). Correo electrónico: sck@jhu.edu

\footnotetext{
Artículo de reflexión

Artículo publicado originalmente en la Revista de Crítica Literaria Latinoamericana 75.XXXIX (2012): 27-50. Documento accesible en línea desde la siguiente dirección: http://revistas.javeriana.edu.co 


\section{Resumen}

Este trabajo detalla la conexión y distinción entre las obras de W. H. Hudson y José María Arguedas, usando la teoría rizomática de Deleuze y Guattari, para evaluar el poder de un paisaje, evocar y crear memorias, $\mathrm{y}$ al mismo tiempo inculcar un ser-sentido. Además, explora el afecto cognitivo chamánico presente en la obra de Arguedas. En cambio, Hudson se centra en la proyección de uno mismo al nivel del individuo. Arguedas muestra un enfoque dual sobre lo cultural y lo individual. Este ensayo está basado en la idea de que hay varios estados de conciencia que influyen en nuestra interpretación de la realidad y nuestra capacidad de sumergir dichos estados en uno de ser-siendo, provocado por el entorno natural descrito por Arguedas y Hudson.

Palabras clave: Arguedas, Hudson, Deleuze y Guattari, rizoma, naturaleza, afecto cognitivo.

Palabras descriptor:Arguedas, José María, 1911-1969, William Henry, 1862-1918, Deleuze, Gilles, 1925-1995, Guattari, Pierre Félix, 1930-1992 - Crítica e interpretación, naturaleza, afecto (Psicología).

\section{Abstract}

This paper describes in detail the connection and distinction between the works of W.H. Hudson and José María Arguedas, using the Deleuze and Guattari's theory of rhizomatic, to assess the power of a landscape, evoke and create memories and, at the same time, instilling a sense-being. Besides, it explores the shamanic cognitive affection present in the Arguedas' work. Instead, Hudson focuses on the projection of oneself to the level of the individual. Arguedas shows a dual focus on the cultural and individual spheres. This essay is based on the idea that there are several states of consciousness that influence our interpretation of reality and our ability to immerse one of these states in a be-being one, caused by the natural environment described by Arguedas and Hudson.

Keywords: Arguedas, Hudson, Deleuze and Guattari, the rhizome, nature, cognitive affection.

Keywords plus: Arguedas, José María, 1911-1969, William

Henry, 1862-1918, Deleuze, Gilles, 1925-1995, Guattari, Pierre Félix, 1930-1992 Interpretation and criticism, nature, affect (psychology).

\section{Resumo}

Este trabalho descreve a conexão e diferenciação entre as obras de W. H. Hudson e José María Arguedas, usando a teoria rizomática de Deleuze e Guattari, para avaliar o poder de uma paisagem, evocar e criar memórias, enquanto um ser-sentido é incutido. Aliás, ele explora o afeto cognitivo xamanista presente na obra de Arguedas. Em vez disso, Hudson centra-se na projeção de si mesmo ao nível do individuo. Arguedas mostra um enfoque dual sobre o cultural e individual. Este ensaio é baseado na ideia de que tem vários estados de consciência que influenciam a nossa interpretação da realidade e capacidade de imergir tais estados entre um de ser-sendo, provocado pelo entorno natural descrito por Arguedas e Hudson.

Palavras-chave: Arguedas, Hudson, Deleuze e Guattari, rizoma, natureza, afeto cognitivo. Palavras-chave descritores: Arguedas, José María, 1911 1969, William Henry, 18621918, Deleuze, Gilles, 19251995, Guattari, Felix Pierre, 1930-1992 - Criticism and interpretation, natureza, afeto (Psicologia).

\section{Cómo citar este artículo:}

Castro-Klarén, Sara. "Recorridos chamánicos: sobre el afecto cognitivo en Arguedas, W. H. Hudson, y Deleuze y Guattari". Cuadernos de Literatura 18.35 (2014): 151-172. 
"I remember-better than any orchard, grove, or wood I have ever entered or seen, do I remember that shady oasis of trees at my new home on the illimitable grassy plain.

This was the world in which I moved and had my being". (Hudson 45, 52)

"Los ríos fueron siempre míos; los arbustos que crecen en las faldas de las montañas, aun las casas de los pequeños pueblos con su tejado rojo cruzado de rayas de cal; los campos azules de alfalfa, las doradas pampas de maíz". (Arguedas 67)

CAMAC, W. H. HUDSON Y ARGUEDAS

LA PRIMERA PARTE de este trabajo se encuentra en su totalidad en "Camac y la memoria emocionada: entrecruces de William Henry Hudson y José María Arguedas", de reciente publicación en Entre Borges y Conrad: estética y territorio en William Henry Hudson (2012), editado por Leila Gómez. En este artículo, dedicado a la exploración de las diferencias y posibles coincidencias entre la teoría rizomática de Deleuze y Guattari y los conocimientos chamánicos o ejercicios de afecto cognitivo en Hudson, tomo en cuenta las tesis y evidencias desarrolladas en el trabajo al cual hago referencia. De hecho, reproduzco aquí algunas páginas de ese primer trabajo, ya que lo que sigue sobre rizomas y chamanismo no tendría sentido completo sin conocer los argumentos y evidencias presentadas en esta primera exploración sobre el afecto cognitivo.

Me encontré con El mundo maravilloso de Henry Hudson (1951) después de estudiar la obra de Julio Cortázar hace ya un buen tiempo. Inmediatamente leí Far Away and Long Ago (1918), libro que me llevó a la lectura de The Purple Land (1885). El orden cronológicamente inverso de mis lecturas en relación con las fechas de publicación de estos libros de Hudson dejó en mi memoria el efecto de una extendida biografía en dos volúmenes, y borró así las distinciones entre esa history of my early life y el carácter débilmente ficticio de The Purple Land that England Lost. Atribuyo esta lectura biográfica, este desliz crítico, este productivo misreading, a un factor predominante en la obra de Hudson, el cual consiste en la evocación consistente y avasalladora de la pampa, no solo como paisaje (landscape), sino más bien como a place of being o ser-siendo. Ofrezco esta formulación de being en inglés para evitar la cortedad de los infinitivos ser o estar en español. Es decir que, como Hudson mismo lo entiende, en una inversión impresionante de los valores de Facundo, civilización o barbarie (1845), la extensión de la ilimitada pampa no es una amenaza, sino que, por el contrario, para la voz infantil que hace memoria, "this was the world in which I moved and had my being, within the limits of the old rat-haunted foss, among the enchanted trees" (Hudson 52-53). 
Creo que esta construcción de la pampa como place of being (ser-siendo) es lo que produce y justifica pensar en una afinidad acronológica con la obra de José María Arguedas. Para ambos hombres la línea de defensa frente al acoso del mundo era el campo, los ríos, algún árbol, un pájaro, un insecto, es decir, ese foss (fosa) afable en donde anidaban las ratas amigas, los sapos semienterrados, los flamencos impasibles al niño que, absorbido en su belleza, los observa en un tiempo fuera del tiempo cotidiano. Ese foss de Hudson metaforiza el sentimiento de convergencia con la naturaleza expresado por Arguedas cuando escribe "yo que sentía tan mío aun lo ajeno" (Arguedas 67).

El gran hallazgo de Hudson, tal como en el caso de Arguedas, es haber creado un lenguaje, pero en especial haber sabido dejar fluir la emoción y la sensibilidad de esa entonces infantil conciencia -ese "far away and long ago"-, ese momento irrecuperable de sensibilidades y convicciones otras a las racionalidades del adulto sobre los accidentes y gentes de la pampa en que su being (ser-siendo) se dio. Es tal vez esta cualidad en la prosa de Hudson la que Borges detecta y,junto con Martínez Estrada, admira y consagra. Comparte Hudson con Arguedas la sensibilidad de los niños solos y errantes; huérfanos accidentalmente liberados de los determinantes de los mayores ${ }^{1}$. Así se reconoce Arguedas en la trémula escena en que despide al padre e imagina los espacios y paisajes que mutuamente se podrían ver y sentir, en los cuales converger, desde el otro lado del abra. La voz del niño se confunde con la situación de forastero del padre. La condición de forastero del padre también pesa en Hudson. Comparte el sentimiento de orfandad con el padre en la Argentina, ya que nunca se aclara la razón por la cual la familia inmigró. Arguedas imagina y se identifica con el sentimiento del padre:

En su calidad de forastero recién llegado, sentiría mi ausencia, yo exploraría, palmo a palmo el gran valle y el pueblo; recibiría la corriente poderosa y triste

1 Aunque ni Hudson ni Arguedas fueron técnicamente huérfanos, ambos comparten la conciencia de la ausencia o del padre o de la madre. Arguedas fue huérfano de madre y sufrió una cierta orfandad en referencia con el padre que no lo tuvo jamás en casa sino que más bien lo dejó con la fría e imperativa madrastra. Hudson tuvo al padre y a la madre en casa, pero el padre aparece siempre como una presencia borrosa, hasta distante y poco directiva. Con la madre sí tuvo una relación afectiva importante. Pero, aunque compartió con ella sus afectos e intereses por el mundo natural y la pampa que rodeaba al hogar, el deambular libre de Hudson por la pampa, el no estar matriculado en ningún colegio y haber crecido como por su cuenta hacen de él una figura comparable al niño y joven Arguedas que busca sus amigos entre los locales, los indios, y sufre más bien un gran choque y desubicación cuando entra al colegio en la adolescencia. El ser arrancado de esa querencia natural no constituye el enfoque de Hudson, quien parece volver a casa cuando se repatria a Inglaterra, pero sí marca la nostalgia del recuerdo por aquella originaria morada de una persona poco tocada por la sociabilidad institucionalizada. 
que golpea a los niños cuando deben enfrentarse solos a un mundo cargado de monstruos y de fuego, y de los grandes ríos que cantan con la música más hermosa al chocar contra las piedras y las islas. (43)

La altísima calidad sensorial del lenguaje, la carga sinestésica de esa "corriente poderosa y triste", entrecruzada con el goce y la alegría de la música de los ríos de Arguedas, es lo que la crítica reconoce en Hudson con el nombre de emoción estética del recuerdo.

La carga visual y olfatoria de un campo de alfalfa en Hudson pudo haber sido cantada a dúo con la voz lírica de Arguedas ante un alfalfar florecido en los Andes. Inmediatamente después de recordar la fosa, feliz escondite de las ratas, en donde él sustenta su being (ser-siendo), y no muy lejos de las mezclas "raras" de la sensibilidad andina de Arguedas (la gozosa y gozada felicidad de las ratas, la transformativa inteligencia de un chancho, la sabiduría de los sapos), Hudson poetiza, es decir, interioriza un alfalfar en la pampa:

There was a field of alfalfa about half an acre in size, which flowered three times a year, and during the flowering time it drew the butterflies from all the sorrounding plain with its luscious bean-like fragance, until the field was full of them, red, black, yellow and white butterflies, fluttering in flocks round every blue spike. (53)

A dúo, Arguedas escribe cuarenta años más tarde, y desde otra vertiente cultural: "Los ríos fueron siempre míos, los campos azules de alfalfa, las doradas pampas de maíz. Pero... al anochecer, se desprendía de mis ojos la maternal imagen del mundo" (67). En ambos amorosos amantes de la "naturaleza" (lo pongo entre comillas por ser un concepto occidental que no abarca la extensión cósmica del ser-siendo andino), la percepción de la luz, el color y la maravilla del espectáculo inunda de goce la escena y su ser-siendo (being). Se da una transferencia, como la del chancho con Arguedas en El zorro de arriba y el zorro de abajo (1971) (véase Castro-Klarén). Rara vez, Hudson registra el momento en que la luz merma, en que la noche borra la imagen en el ojo, en que la tristeza y la melancolía arrancan del recuerdo su gran alivio y consuelo, como es casi siempre el caso en Arguedas.

Salvando la raigambre europea del pensamiento científico-sensible en Hudson, nada más y nada menos se podría decir, y en algunos casos ya se ha dicho, que el pensamiento andino tiene una concepción de la naturaleza que difiere de la europea, pero no por eso es (fue) menos inteligente o "científica", es decir, sistemática, atenta al más mínimo detalle y experimental en sus observaciones. Son estos saberes andinos, en los que la información recogida por los aparatos 
sensoriales no rechaza la afectividad del momento cognitivo, sino que más bien la busca a manera de complemento y/o meollo, los que aparecen en las escenas naturalistas de Hudson y en las tomas de las retamas colgantes, los eucaliptos y las bandas de loros en el mundo de Arguedas. Esta aproximación andina, a veces tildada de chamánica, en la que se busca la compenetración (convergencia) con una espiritualidad que se da en niveles de conocimiento otros en relación con la conciencia de lo cotidiano occidental, es la que informa sobre la sensibilidad particular de la prosa de José María Arguedas. A su vez, es la plasmación de estos otros niveles de conocimiento sensible lo que permiten o, más bien, invita a una comparación entre la mirada o el sentir de Hudson y el del autor de Los ríos profundos (1958). La presente aproximación comparativa no intenta igualarlos. Más bien, trata de investigar las dimensiones hasta ahora no elucidadas de las pertenencias y diferencias que les confieren su lugar único en el canon de la literatura moderna.

En "Como chancho cuando piensa" (2001) estudié las dimensiones y dinámicas del afecto cognitivo en El zorro de arriba y el zorro de abajo (1971). Tomando como paradigma la escena en que el narrador cavila sobre su deseo de llegar a "ser", o más bien transformarse en "un chancho cuando piensa", llevé adelante la idea de que Arguedas opera no solo en esta escena, sino en muchos otros momentos de su escritura, una transvaluación, una operación de transvaciamiento que permite accesos de conciencia entre una especie y otra. Estos accesos interespecie constituyen un aspecto clave del chamanismo del que Gilles Deleuze y Felix Guattari intentan apropiarse y desplegar en A Thousand Plateaus: Capitalism and Schizophrenia (1987) para su teoría rizomática, cuando hablan de estados de conciencia que constituyen un becoming, aunque, como veremos más adelante, más bien distorsionan con su concepto de rizoma y su énfasis en la chatura de los devenires.

Ser, actuar, sentirse o ser-siendo como chancho cuando piensa, en cuanto planteamiento que elucide esa característica única e indeleble de la prosa naturalista de Hudson o las evocaciones de campos, personas o montañas en Arguedas, no apoya la tesis de Martínez Estrada respecto al hallazgo de una aproximación científica en Hudson y ausente en los escritores argentinos. Esta idea de un acceso de tipo chamánico a otros estados de conciencia sí sustenta la intuición del autor de Radiografía de la pampa cuando apunta a otra raigambre en el pensar-sentir de Hudson. Esa raigambre no es la ciencia ni, claro está, el empirismo, ni menos el realismo de la novela regional. Es algo diferente, una mezcla, más arguediana, es decir, más cercana al afecto cognitivo chamánico y menos cultivada en el adentro de los marcos clasificatorios de la tradición europea. 
En mi estudio sobre lo que podría significar "pensar como chancho" en Arguedas, demuestro que lo que ocurre en esa escena en El zorro de arriba y el zorro de abajo y repercute a través de la posibilidad/imposibilidad de la escritura de la novela/autobiografía, es el registro de "una conciencia afectada por la expansión, ocupación y contagio con la multiplicidad" del cosmos (Castro-Klarén 34). Esta multiplicidad es alcanzada por el cámac, por un estado o modo de ser (being), el ser-siendo de alma-cuerpo (31) que constituye el meollo de la concepción del cosmos andino. Cámac no corresponde a la división oposicional racional/irracional de Occidente ${ }^{2}$. Es la plasmación lingüística de ese contagio, ese transvaciamiento mutuo entre hombre e "immensely tall, white and rose-colored birds" (78), entre el niño y "the sweet green silences" (111), entre William Henry y el "wild solitary spot where I could spend hours watching the birds" (54), o entre el errante muchacho y "the sacred flowers" de la retama (228) en Far Away and Long Ago, lo que cautiva la imaginación y los afectos de Borges y Martínez Estrada por la pampa de Hudson.

Habiendo entrado en un estado de conciencia no-ordinario, similar al del chamán, Hudson pone su cámac diariamente en sus recorridos sin rumbo en la ilimitada pampa y en sus días perdidos entre la visión física y la ensoñación de la planicie sin fin que se le produce en la conciencia. Años después, recupera ese estado de conciencia contaminada en las páginas en las que textualiza la memoria. Por su parte, Arguedas lucha contra el olvido y la borradura de la memoria de sus vivencias infantiles, de sus momentos de cámac, desde sus primeros cuentos en los que se propone contarlo "tal cual es" y no tergiversado por los parámetros y usos del castellano. Después de la lectura remecedora de los mitos de Huarochiri, vislumbra que cuando el cuerpo-alma se pone a trabajar, lo que se produce es un (peligroso) pachacuti, un convertirse en animal, en dulce verde, en tierno arroyo, en chancho. Estos errantes niños, en su abandono y libertad, viajan por varios y múltiples estados de conciencia. Esa errancia anclada en su being (ser-siendo), en la "maternal imagen del mundo" (Arguedas 67), les permite escapar de los sedimentos y límites de la conciencia práctica de la vida burguesa.

A esos estados de conciencia Hudson los llama animismo, y Arguedas los conoce como la concepción-sentimiento del cuerpo-alma andina. Arguedas, tal y como rechaza el apelativo de transculturación para su being y sus saberes y artes, rechazaría plenamente la idea de que los estados de conciencia, la aprehensión

2 Véase al respecto el análisis de cámac de Frank Salomon en su introducción a su edición del Manuscrito de Huarochirí. 
del mundo que busca plasmar en español a pesar de las deficiencias de este idioma para la tarea del cámac, podrían ser descritos con el término animismo.

En cuanto concepto que viene de la antropología jerarquizante del siglo XIX, además de ser inexacto, para Arguedas sería reconocido como parte de la máquina arrolladora de denigración de las culturas indígenas americanas. Animismo hubiera recibido el pleno rechazo de Arguedas. En vista de toda la crítica actual al eurocentrismo, es claro que hoy en día tampoco sirve ese concepto. Pero Hudson lo aceptó en su tiempo, porque en parte no había otra cosa, y el maduro naturalista adscribía ese estado de conciencia a su infancia, como una manera de encontrar alguna explicación que tuviera sentido dentro de los marcos epistemológicos en su entorno. Por medio de las letras de entonces, el argentino nada o poco sabía, o podía haber sabido, de los estados de conciencia chamánicos.

En el capítulo "A Boy's Animism" de Far Away and Long Ago, Hudson busca una explicación a la calidad afectiva de sus indelebles memorias. La memoria de una culebra negra molesta la reconfortante presencia de otras memorias más tiernas y amables. "These serpent memories, particularly the enduring image of that black serpent which when recalled restores most vividly the emotion experienced at the time" (224). Hudson dice que esa operación de la subjetividad en la que surgen tanto la poderosa evocación de la imagen como la vivencia repetida de la emoción primera se llama animismo:

This is animism, or that sense that something in nature which to the enlightened or civilized man is not there, and in the civilized man's child, if it admitted that he has it at all, is but a faint survival of a phase of the primitive mind. (224)

No es, pues, de extrañar que tanto Borges como Martínez Estrada avanzaran en la sugerencia y argumentos de Hudson sobre su animismo y lo llamaran el gran primitivo. El gran primitivo en boca de Borges se enriquece con las alusiones a Whitman, considerado como el "amistoso y elocuente salvaje" de Leaves of Grass (1855) ("Nota sobre Walt Whitman", en Otras inquisiciones 99), con las miradas al conocido panteísmo del compatriota de Hudson. Como bien observa Leila Gómez, "tanto en el campo intelectual inglés como en el rioplatense, Hudson será conocido como 'el gran primitivo' (Manning 1941) y en eso consistirá su capital simbólico" (44).

No obstante, Hudson oscila entre distanciar y abrazar las supervivencias de la mente primitiva de sus capacidades perceptivas, a las que prefiere llamar animismo. Distingue entre la "theory that there is a soul in nature" (las creencias de la mente primitiva) y la "tendency or impulse or instinct, in which all myth originates, to animate all things; the projection of ourselves into nature; the sense 
and apprehension of an intelligence like our own but more powerful in all visible things" (224-225; el énfasis es propio). Esta apreciación de Hudson -"the projection of ourselves into nature" - está mucho más cerca de la experiencia del chamán o del saber afectivo andino que del animismo. La explicación de Hudson, sin embargo, no deja de ser teológica a la manera tomística. Es decir, que en una operación de proyección del ser humano percibimos el orden y la perfección divina que Dios pone en la naturaleza de la cual formamos parte. En este sentido, el animismo de Hudson roza con el deísmo rousseauniano de Whitman y difiere así de la relación andina evocada por Arguedas. En la ambición arguediana de "pensar como chancho" no se borra la diferencia con lo otro. Es un convertirse (no en el otro, al estilo de Ovidio), sino con el otro (mezclarse, entrelazarse sin hibridarse, diría Arguedas). El momento arguediano es ateológico, tal y como lo fue la religión (valga la paradoja) andina. El alma, the soul, de la que habla Hudson, no es comparable al alma-cuerpo del cámac.

A pesar de la diferencia entre el cámac y el alma, creo que Hudson, sin otros medios intelectuales a su alcance en la época en que la antropología de sir Edward Tylor había inventado el animismo como recurso para entender las religiones "primitivas", cuando abraza el término primitive, para referirse a sus estados de afecto cognitivo, habla no del animismo de Tylor, sino más bien de algo parecido al momento cámac de Arguedas. Hudson vive admirado de no haber perdido su "animismo" a pesar de que en su primera tesis sobre el asunto lo atribuyera a los efectos ambientales de vivir "in rural sorroundings, where there are hills and woods and rocks and streams and walterfalls, these being the conditions that are most favorable to it" (225). Ya viejo, en Inglaterra, y haciendo memoria para escribir Far Away and Long Ago, se encuentra pensando:

I know that in me, old as I am, this same primitive faculty which manifested itself in my early boyhood, still persists, and in those early years was so powerful that I am almost afraid to say how deeply I was moved by it. (225)

Ese miedo irresistible, porque viene mezclado con una "ternura feroz" (véase la "Nota sobre Walt Whitman" de Borges, en Otras inquisiciones 101), es la dimensión afectivo-cognitiva que enlaza a Hudson con Arguedas, a pesar de que en Hudson es únicamente individual y en Arguedas es individual y cultural. $\mathrm{Al}$ respecto, Borges observa con agudeza muy propia que lo indispensable en Whitman no es el deseo de ser y hablar todo y por todos, sino más bien la insólita calidad de la memoria que plasma los bosques de América en el poeta norteño. En "El otro Whitman", Borges descubre, al citar al poeta según va escribiendo la corta nota, que la singular marca del poeta es la "simplificación final del 
recuerdo, inconocibilidad y pudor de nuestro vivir, negación de los esquemas intelectuales y aprecio de las noticias primarias de los sentidos" (208). Hago aquí este descubrimiento extenso a Hudson, a ese Hudson que supo, en la figura de Richard Lamb, "recibir todas las vicisitudes del ser, amigas o aciagas" ("Sobre 'The Purple Land", en Borges, Otras inquisiciones 198). Es este encuentro con el esplendor de inconocibilidad de las cosas, incluso de nosotros mismos, el recibimiento de todas las vicisitudes del ser y del acaecer, el que anima la prosa de Hudson y lo emparenta con Arguedas. El parentesco Hudson-Arguedas emula el momento chancho cuando piensa, iluminado, paradójicamente por momentos de inconocibilidad para la razón de occidente, pero sí accesibles al afecto-cognitivo, a la travesía en el campo interespecie.

\section{Rizomas, pampas y chamanismo}

En mi artículo sobre la convergencia entre el ser-siendo (ofrezco esta rendición de being) animal/humano, sobre el pensar como chancho, estadio al cual Arguedas aspira en El zorro de arriba y el zorro de abajo, presté intensa atención a la exploración afectiva que se da en esa novela/diario, como también a la discusión teórica de Deleuze y Guattari en A Thousand Plateaus: Capitalism and Schizophrenia en torno a la idea de becomings o momentos de intensidad. En esa discusión me di cuenta de que los becomings (devenir, en el original francés y en la traducción al español) que Deleuze y Guattari teorizan a través de la teoría de los afectos de Baruch Spinoza (xiii, 153-154, 256-257, 260-261) demandan una genealogía en sí, antes de ser puestos en circulación como matriz de comprensión de los momentos de afecto cognitivo en Arguedas o Hudson. En una primera lectura el concepto de becomings parecería tener mucho en común con las convergencias intraespecie de ser/siendo en Arguedas. Pero las lecturas repetidas de Arguedas y de $A$ Thousand Plateaus me han dejado con dudas.

Es más, una consideración más enfocada en la raigambre andina de las cosas, ausente en toda la obra de Deleuze y Guattari, provoca un cuestionamiento que va no solo mucho más allá de la propuesta rizomática de los teóricos franceses, sino incluso de otras inimaginadas e incomibles direcciones. Hablo de inimaginadas e incomibles direcciones, ya que a pesar de todo el esfuerzo por discurrir desde un afuera nómada al pensamiento y la filosofía de Estado que Deleuze y Guattari muy justamente critican, su debate apunta siempre a conversaciones polémicas con el psicoanálisis, ya sea este freudiano o lacaniano. La inevitable y dominante conversación con el psicoanálisis constituye una fuerza extraordinaria que tira, afloja, resquebraja y corruga cada una de las mesetas (pampas, diríamos nosotros) o superficies lisas, de manera tal que las amargas polémicas con Freud, 
Melanie Klein, Lacan y otros se interponen en toda consideración de la cuestión del becoming o devenir animal o vegetal. Uno de los mejores ejemplos de esta gravitación hacia la muy justa polémica con el psicoanálisis se da en el capítulo segundo, en el que Deleuze y Guattari presentan por primera vez la cuestión intraespecie en el caso del wolfman en el capítulo titulado "One or Several Wolves". Deleuze y Guattari le reprochan dura y amargamente a Freud el reducir todo a sus esquemas edípicos y sexuales con la subsiguiente incapacitación y censura del sujeto bajo terapia:

During the first episode, which Freud declares neurotic, [the wolfman] recounted a dream that had six or seven wolves in a tree, and drew five. Who is ignorant of the fact that wolves travel in packs? Only Freud. Every child knows it. Not Freud... He had decided that this was neurosis, so he uses the other reductive procedure: free association on the level of representation of things, rather than verbal subsumption on the level of the representation of words. The result is the same... the wolves will have to be purged from their multiplicity... Who is Freud trying to fool? The wolves never had a chance to get away and save their pack: it was already decided from the very beginning that animals could serve only to represent coitus between parents... Freud obviously knows nothing... Freud only knows the Oedipalized wolf or dog, the castrated-castrating daddy-wolf, the dog in the kennel, the analyst's bow-bow. (28-29)

Vale la larga cita ya que no solo manifiesta la crítica al método reductivo y la obstinada teoría edípica de Freud, sino que también establece el tono sardónico de Deleuze y Guattari en relación con las escuelas freudianas en general. Esta crítica al método y la teoría freudiana no debe ser olvidada en relación con nuestras posibles lecturas (no-reductivas) de los cosmos amerindios. Es importante hacer hincapié al respecto, y en especial en relación con los Zorros en cuanto, como ya lo he sugerido, Arguedas estaba recibiendo tratamiento de una psicoanalista freudiana durante la crisis en que se imbrica la escritura de la novela/diario.

Es más, en la consideración de los becomings o devenires que Deleuze y Guattari teorizan no es posible olvidar que la idea central de A Thousand Plateaus se inspira en la lectura de los estudios sobre la cultura balinesa de Gregory Bateson $(21-22,158)$ y en sus observaciones sobre ciertos momentos y rituales de intensidad en esa cultura. Así, pues, poco o nada tienen que ver con lecturas sobre culturas amerindias, aunque ambas, en sus distintos tiempo-espacios, viven fuera del espacio-tiempo de Occidente, y por lo tanto se administran fuera o sin relación necesaria con los estadios edípicos de aquel Freud que no sabe 
nada de lobos y menos de hombres-lobos o lobisones. Brian Massumi, en el prólogo a la edición en inglés de Mil mesetas, advierte que Bateson encontró en la cultura balinesa "a libidinal economy quite different from the West's orgasmic orientation" (xiv). En la introducción se establece que los rizomas están hechos de mesetas. Este concepto es también tomado de Bateson, para quien meseta (plateau) designa algo muy especial, es decir, "a continuous self-vibrating region of intensities whose development avoid any orientation toward a culminating point or external end" (22-23). Así, pues, una meseta sería "any multiplicity connected to other multiplicities by superficial underground stems in such a way as to form or extend rhizomes" (23). De manera que, conceptualmente, no se podría separar meseta, una planicie de intensidades, de rizoma. Como tampoco se puede optar por adoptar la idea de becoming animal, sin tener en cuenta que con ello se importa también todo el complejo rizoma y su oposición a la filosofía de Estado en que Deleuze y Guattari construyen y desarrollan estos conceptos.

Nos incumbe, pues, un pequeño recorrido sobre la hechura del rizoma, y en especial el concepto spinoziano de afecto, para ver hasta qué grado es compatible o capaz de iluminar lo andino, porque bien puede ser que el afecto cognitivo andino y los estados de devenir-intenso no sean comparables, afines, paralelos ni menos partícipes de un mismo estado de conciencia o prácticas. Por el contrario, podría ser que el tema de la compaginación fuera de serie de la orquídea y la avispa (Deleuze y Guattari 10) sí tenga parecidos con las convergencias andinas, como el huayronqo y la flor zapatilla. La flor zapatilla incomoda, creo, una serie spinoziana, y más aún una serie freudiana, si atendemos a la discusión de Deleuze y Guattari. Por si acaso, queda sin más comentario, en esta discusión de convergir, el afirmar con Deleuze y Guattari que el concepto de mimicry no solo es inútil para pensar en la flor-zapatilla o la orquídea-avispa, sino "a very bad concept" en general $(11,236)$.

En vez de pasar por Deleuze y Guattari y su productiva elaboración del concepto de plateau e intensidad tomados de Bateson, sería más provechoso leer directamente a Bateson y su descripción de las mesetas de intensidad balinesas, para así compararlas directamente con lo que hasta el momento constituye el cosmos andino o el concepto de camac -animador del mundo, o Pachamama-. Es más, Massumi señala que la economía libidinal balinesa es bastante diferente en cuanto no aparece orientada orgásmicamente al estilo de Occidente en Freud (xiv). Esta comparación directa se hace más urgente para evitar pasar por todo el circuito de la polémica con el psicoanálisis en Deleuze y Guattari en la que, a pesar de sus esfuerzos, queda enterrada y hasta enraizada su propia elaboración del concepto rizoma como antídoto de los problemas ocasionados por la imagen 
raíz. Sin embargo, es el poder del pensamiento de Deleuze y Guattari en nuestros medios discursivos y no el libro de Bateson el que provoca esta discusión respecto a la cuestión de devenires o convergencias intraespecie en Arguedas o Hudson y, por lo tanto, demanda algunas elucidaciones de términos y conceptos.

Ligado como está el concepto de meseta (plateau) al de rizoma, y en vista de que a su vez ambos engarzan con el de nomadía, es importante recalcar aquí la situación circunstancial, diríamos casi eventual, de un becoming o devenir de intensidad. A este respecto es útil notar la aclaración hecha por Massumi en el prólogo a la edición en inglés: "A Plateau is reached when circumstances combine to bring activity to a pitch of intensity that is not automatically dissipated in a climax" (xiv). Esta cumbre de intensidad crea a su vez una red de posibilidades y conexiones con otras rutas y puntos de escape (xiv). Massumi reitera que el propósito de la escritura del One Thousand Plateaus, libro fasciculado en sí, es provocar o construir esas mesetas de intensidad para pensar en una nueva dinámica a la que Deleuze y Guattari llaman pragmatics (xv). El objetivo de tal pragmatics es la invención de conceptos resistentes a la sistematización en contra de la cual escriben (xv). O sea que el libro mismo, tal vez, como los Zorros, sería un devenir, una experiencia de un becoming, una intensidad inusitada y que excede todos los géneros y los sistemas. Sin embargo, es evidente que con solo prestar atención a los redoblados y sistemáticos objetivos del pensamiento de Deleuze y Guattari -proponer el concepto no-organizador de rizoma en contraste y en sustitución de los conceptos sistematizadores basados en la metáfora arbórea que preside la historia del pensamiento occidental- quedamos a gran distancia de los sueltos y únicos momentos de afecto cognitivo o conciencia chamánica que se plasman en la prosa de Arguedas y Hudson, muy de vez en cuando aunque sí embebidos de gran intensidad.

Teniendo en cuenta un parámetro comparativo, vale aquí la pena volver brevemente sobre algunas de las características del rizoma para así examinar los posibles puntos de contacto entre un devenir o becoming y un momento de convergencia intraespecie, como se daría en Arguedas o Hudson. La primera característica es el modelo del bulbo y el rechazo al de la raíz. Es importante recordar aquí que el modelo visual trata de redes relativamente superficiales, en oposición a raíces profundas. Es notable que Deleuze y Guattari no hablan de la papa, el bulbo andino cuyo valor alimenticio transformó la historia del mundo y cuya confección de ojos y raíces se presta mejor a la imagen del rizoma que el bulbo del barbudo tulipán, por así decirlo. Lo que interesa aquí es la imagen de la conectividad subterránea, aunque superficial, de los tendriles y raíces del bulbo, y el salto conceptual que Deleuze y Guattari dan con este modelo visual 
a los rizomas y las mesetas, a pesar del hecho de que cada bulbo vive y se reproduce única e individualmente, sin necesidad de conexión con ningún otro bulbo. Segundo, no es menos interesante observar el otro salto en cuanto al rizoma de conectividad y las colectividades animales que les sirven de segundo modelo y punto de reflexión: las colonias de hormigas, los panales de abejas, los enjambres de insectos y las manadas de lobos y ratas (7). El énfasis en esta conceptualización del rizoma recae sobre la conectividad a través de la heterogeneidad, pero lo que resume y destila de estas metáforas e imágenes vegetales y animales es más bien la conectividad dada en una cierta homogeneidad: enjambre de ratas-ratas, manada de lobos-lobos. No es hasta el ejemplo de la orquídea con la avispa (10) (tercera característica) que nos encontramos ante un cierto devenir intraespecie. Mientras que en los casos de Arguedas y Hudson los encuentros y convergencias entre especies, tipo hombre-lobo, ratas-niño, son típicas, en Deleuze y Guattari se limitan al hombre-lobo y la orquídea-avispa. Es pues la tercera característica del rizoma -la multiplicidad heterogénea-, o sea la orquídea-avispa, la que ofrece mejores posibilidades de contacto con el converger del chamán. El complejo orquídeaavispa sería comparable al complejo huayronqo-flor zapatilla en Arguedas, o la fosa de ratas-niño en Hudson.

Para Deleuze y Guattari, el cuarto principio, el de multiplicidad, es clave en la resistencia del rizoma a principios de unidad o sistematización. No sé hasta qué punto esta prescrita resistencia a los principios de unificación podría encontrar ecos o paralelos en la cosmología andina, que más bien se empeña en la reciprocidad y las correspondencias geométricas finitas y cerradas. Pero en cuanto la multiplicidad del rizoma ocasiona que "there is no unit to serve as pivot of the object... multiplicity has neither subject nor object" (8), podríamos decir que el conjunto heterogéneo orquídea-avispa lleva a pensar que, al mantener separados pero en conjunción sus dos términos, acusa puntos de afinidad con el deseo de habitar la zona de "como un chancho, cuando piensa". En este converger, como ya lo dije en mi artículo sobre los Zorros, no hay un devenir del uno en el otro o de los dos términos en un tercer punto unificador: la multiplicidad aquí se manifiesta más bien en un abolir de la relación sujeto-objeto, como lo postulan los filósofos franceses. No se trata para nada, en ninguno de los casos, de una unidad mística. Nada tiene que ver esta figura con el animismo tampoco.

Por el contrario, son los conceptos andinos de la reciprocidad, y del hanan y el hurin, en los cuales la relación es de copresencia, de convergencia, sin jerarquía sujeto-objeto, los que corresponden a la iluminación de este tipo de multiplicidad. Así, pues, siguiendo el pensamiento andino, una convergencia chamánica, como en el mejor caso del rizoma, no admite ser overcoded (9) ni sistematizada 
y menos comodificada. Es siempre una intensidad única, eventual y múltiple, y corresponde a las coordenadas del orden de los saberes-cognitivos chamánicos. Es importante observar que Deleuze y Guattari casi no prestan ninguna atención a los saberes-cognitivos chamánicos en el largo capítulo "Becoming Intense", en el que abundan sobre el hombre-lobo, el hombre-rata y toda su teoría de los becomings. En un corto párrafo sobre brujos (sorcerers) se alude a Carlos Castaneda, aunque don Juan y sus transformaciones en animales quedan fuera de la discusión. Paso ahora a una corta discusión de lo que llamo afecto-cognitivo en algunas experiencias chamánicas, que pueden tener una relación más cercana con Arguedas y Hudson, ya que en ellas se trata de conectar o converger con el nahual o power animal en una búsqueda de equilibrio o bienestar perdido, tal como en el caso de muchos de los textos de Arguedas.

\section{Arguedas, Hudson, chamán: aprendiendo de los árboles}

Al terminar mi trabajo sobre Hudson y Arguedas, leí la colaboración de Ricardo Salvatore para el volumen sobre Hudson y Borges editado por Leila Gómez, y para el cual escribí ese trabajo. Salvatore confirmó una impresión que me iba quedando de la crítica, en especial la de Borges, sobre Hudson, y es la innegable ausencia de todo rastro de las culturas o agentes de las culturas indígenas de la pampa con las que Hudson tuvo o pudo haber tenido contacto. En ese momento quise volver sobre lo que había investigado, releer a Hudson a ver qué rastros podrían ser detectados en su "sentir" de la naturaleza, pero era ya demasiando tarde. El trabajo hubiera excedido las páginas que se me pedían. En el entretanto seguía cavilando sobre la relación de Arguedas con los saberes chamánicos andinos; con los zorros, los chanchos, los loros, las piedras y los ríos, asunto que había tratado con anterioridad respecto de El zorro de arriba y el zorro de abajo (1971). Mi estudio sobre la relación con la deseada chanchedad intentó sostener la idea de que en la tradición andina existe una dimensión de ser que reside en un espacio donde se da un devenir, un convertirse, no en otro, sino en una extensión conjunta y alterna al mismo tiempo, en un "animal", es decir, en una dimensión de ser-siendo (being), que excede pero no contradice la unidad de la experiencia cotidiana. Esta operación de conversión dentro del ser-siendo (o mejor, del habitar/morar) podría constituir algo parecido a lo que Deleuze y Guattari teorizan bajo el rubro de becoming.

Para ese estudio sobre El zorro no alcancé a entrar en la cuestión de la cultura chamánica y su presencia o ausencia en Arguedas, a pesar de que todos hemos dicho tanto de las coordenadas y la indeleble presencia del "cosmos andino" en los textos arguedianos. Es evidente que es posible encontrar más figuraciones y 
presencias de las culturas chamánicas en Arguedas que en Hudson. Aquí decidí emprender el estudio de lo que sigue.

Con esos resultados en mente recorrí el libro de Michael Harner, The Way of the Shaman: A Guide to Power and Healing (1979). Le di preferencia al libro de Harner, sobre muchos otros, en especial sobre el famoso libro de Mircea Eliade, el de Deleuze y Guattari y el de Michael Taussig, porque entre todos ellos Harner es el único que escribe desde su posición de aprendiz y maestro chamán. No hay nada parecido sobre el chamanismo en los Andes. Harner escribe desde la práctica de un hombre occidental aprendiz del chamanismo jíbaro para la práctica de personas "ordinarias" ubicadas culturalmente en Euroamérica. Es decir que los saberes de Harner pretenden no ser especulativos, sino más bien una reflexión basada completamente en la experiencia empírica y guiada por maestros jíbaros y conibas. No teoriza en lo más mínimo. Me interesa Harner porque no opta por la senda del misticismo, como lo han hecho otros estudiosos del chamanismo. Rechaza este tipo de aproximación que coloca los saberes chamánicos en un más allá o un más acá de la razón y del orden ordinario de las cosas. En este sentido, Harner, sin ser explícito al respecto, coincide conmigo en la idea de que la "religión" andina, el "culto" a las huacas, las colecciones de conopas son una conjunción de saberes experienciales sui géneris que excede la idea occidental de religión, y que por lo tanto debe ser abordada desde una perspectiva basada en la experiencia y no en conceptos tales como trance místico, ya que la idea de lo místico se basa en el concepto de un Dios judeocristiano que es por excelencia otro.

En lo sigue haré algo muy simple. Se trata de un recorrido por los principales hitos que en conjunto constituyen la práctica del chamanismo jíbaro, tal como la presenta Harner, para así invitarnos a la reflexión sobre la obra de Arguedas y la presencia del chamanismo en su obra/vida. Empecemos por una definición que nos aproxime a la antigüedad y las características de los saberes chamánicos: "Shamans are the keepers of a remarkable body of ancient techniques that they use to achieve and maintain well-being and healing for themselves and members of their communities. Their methods are strikingly similar the world over" (xi). Basado en una investigación hecha sobre saberes chamánicos en varias zonas del globo, Harner establece: "through trial and error ancient people arrived at the same conclusions" (xi). Queda, pues, bien claro que los saberes y prácticas chamánicas se fundamentan en prácticas experienciales (pragmatics), que poco o nada tienen que ver con los razonamientos y teorizaciones que se dan en los esfuerzos de Deleuze y Guattari por establecer no solo el rizoma, sino las prácticas de las mesetas de intensidad. 
El chamán es primordialmente un miembro de una comunidad. No es un individuo que flota en una sociedad posmoderna nómada. El chamán está profundamente integrado en la comunidad, su cultura y los intereses que definen su supervivencia. Entre ellos ocupa un lugar importante la salud del chamán y la de otros miembros de la comunidad, para quienes el concepto de salud descansa en la indisoluble unidad del cuerpo y el espíritu. Por lo tanto, el chamán es el curandero por excelencia. Tanto el hombre como la mujer investidos con los poderes afecto-cognitivos del oficio de chamán son personas indispensables en una comunidad que se refleja en las habilidades y los poderes curativos del sus chamanes. La mutualidad es la relación más importante entre los miembros de la comunidad en la que un chamán funciona. Los chamanes practican sus poderes/saberes curativos por medio de viajes a dimensiones diferentes, pero no extrañas o contrarias a las dimensiones fenomenológicas de la vida diaria. No son sacerdotes. Harner advierte:

[...] through heroic journey and efforts the shaman helps his patients transcend their normal, ordinary definition of reality, including the definition of themselves as ill. The shaman shows his patients that they are NOT EMOTIONALLY AND SPIRITUALLY ALONE in their struggles against illness and death. (xi)

El chamán, a diferencia del pragmatics del rizoma, viaja a un lugar muy interno e interior en su propia conciencia, como también a la conciencia del paciente, para rescatar una experiencia perdida u olvidada. Se trata, pues, de asumir una profundidad interior muy a diferencia de la chatura y especialidad del rizoma y sus conectividades. Lo que se da entre el paciente y el chamán es una especie de convergencia en cuanto "the shaman shares his special powers and convinces his patients, on a DEEP LEVEL OF CONSCIOUS-NESS, that another human is willing to offer his own self to help them" (xi). Esta disposición al sacrificio y la convergencia con el otro se hace presente en muchos momentos en la obra de Arguedas -el pongo en la casa del Viejo, los loros en los huertos de Abancay, Palacitos, la Opa, Moncada, el mismo Arguedas en El zorro-. La mutualidad del converger es impresionante en la carga emotiva que la sostiene y la constituye: "The shaman's SELF-SACRIFICE calls for a commensurate emotional commitment from the patient" (xi). No sabría decir hasta qué punto este converger acusa paralelismos con las mesetas de intensidad de Deleuze y Guattari, pero a primera vista se diría que se trata de dos experiencias y dos objetivos que poco o nada tienen en común.

En armonía con el concepto de curar a otro miembro de la comunidad y compartir el conocimiento del ser-siendo en la otra dimensión a la cual es 
necesario acceder para encontrar la sanación, el libro de Harner se escribe con la intención de hacer llegar estas prácticas y conocimientos al mundo occidental que carece de estos. Se trata de compartir, no de jerarquizar; de extender un aprendizaje para rescatar la habilidad de viajar en el espacio y el tiempo interespecie, de "aprender de los árboles" ("learn from the trees"), tal y como le enseñaron a él los conibos en el alto Amazonas (xii).

A diferencia del rizoma, lo que cuenta en este aprendizaje y en este viaje son los poderes personales del curandero y el dominio que él ejerce sobre las técnicas que aprendió de su maestro. Así, pues, el libro de Harner dedica una buena parte no solo a la descripción de las técnicas del viaje a la otra dimensión, sino que desarrolla todo un programa de instrucciones que el lector, con la ayuda de un compañero, puede poner en práctica para así acceder a esa otra dimensión que está siempre presente aunque no actualizada. Es este sentido, el chamanismo descrito por Harner y practicado por Arguedas o Hudson difiere sustancialmente del discurso más o menos siempre teórico y dirigido al psicoanálisis de Deleuze y Guattari. Es más, las simples y poderosas técnicas del chamán parecerían diferir sustancialmente de cualquier cosa identificable por el psicoanálisis, e incluso de la teoría rizomática de los becomings. A este respecto Harner advierte:

For his fellows tribesmen the Jivaro does not need to specify in which state of consciousness he was in to have a particular experience. They immediately know because they have already learned what kinds of experience occur in the SSC and what kind occur in the OSC. (48)

En cuanto aprendiz de la tradición jíbara y estudioso del chamanismo -aspecto ausente en $A$ Thousand Plateaus - , Harner asegura:

$[\ldots]$ the Jivaro sophistication is far from unique; in fact, it is probably true throughout virtually all of the shamanic cultures. Unfortunately Western observers, lacking extensive experience with altered states of consciousness, altogether too often failed to inquire as to the cognitive state in which their native informants were when they had "impossible" experiences. (48)

La experiencia chamánica se da en y trata espacios diferentes, pero contiguos; compartidos, pero separados. Harner explica:

In engaging in shamanic practice, one moves between, what I term an ordinary state of consciousness (OSC) and a shamanic state of consciousness (SSC). These states of consciousness are keys to understanding, for example, how Carlos Castañeda can speak of an ordinary reality and a non-ordinary reality. 
The difference in these states of consciousness can perhaps be illustrated by referring to animals. Dragons, griffins and other animals that would be considered as "mythical" by us in the OSC are "real" in the SSC. The idea that there are "mythical" animals is a useful and valid cons-truct in OSC life, but superfluous and irrelevant in SSC experience... The shaman has the advantage of being able to move between both states of consciousness at will. (xiii)

No se trata, pues, de fantasía. Para el chamán ambos estados de conciencia son igualmente reales. A mi modo de ver, esta doble y armoniosa conciencia es lo que Arguedas busca y a veces consigue plasmar en su prosa y su canto a la naturaleza. Algo parecido podría decirse de Hudson cuando, absorto ante una laguna o la fosa de las ratas, o un árbol, consigue entrar en esa otra dimensión de goce y encuentro.

Mientras que en algunas culturas la entrada al estado de conciencia no-ordinario se da por medio del consumo de drogas, casi universalmente, el acceso al estado de conciencia no-ordinario depende de la voluntad del chamán (xiv). Esta experiencia comparte algunos aspectos con el estado de conciencia que en Occidente conocemos como éxtasis, ya que la experiencia del chamán es siempre positiva (xvi). Harner narra su viaje al mundo-espíritu (spirit world) en su primer aprendizaje con los jíbaros, el cual incluyó la aterradora experiencia del consumo de ayahuasca (7). Pero no se trata de consumir ayahuasca y entrar en el mundo-espíritu, sino más bien de prepararse, a través de una purga física y espiritual, para el peregrinaje necesario a las remotas cascadas de agua, lugar de entrada al mundo-espíritu (13). El espacio natural del mundo de los jíbaros y otros practicantes del chamanismo se encuentra dotado de lugares especiales, o entradas, al mundo-espíritu. Entre estos lugares de acceso, tal como en los Andes, las lagunas, las cuevas, los huecos en los árboles, las cascadas y las grandes rocas constituyen los lugares de peregrinaje para ingresar a esa otra dimensión. A menudo estos lugares "sacralizados" por la práctica chamánica son espacios en los que también se dan fenómenos luminosos o auditivos. La música de las cascadas, el oleaje del mar, el estallido de las olas contra una gran roca, el zumbayllu y el rumor de una arboleda (huacas) son todos fenómenos experienciales que asisten en el viaje a ese otro estado de conciencia. Tal como en Arguedas, en Hudson, para los jíbaros el concierto de la inmensa caída de agua de su cascada sagrada facilita la entrada al mundo-espíritu. Harner recuerda que: "The sound of the cascading water... I left that this is where I belonged, that I had come home" (14). Arguedas a menudo dice sentirse en casa, en el lugar de su pertenencia, cuando llega a la orilla de un cantor río andino, o se siente cobijado por 
el rumor de un ojo de agua, o próximo a un río hablador. Estos grandes árboles y cascadas, protegen a Arguedas igual que a los peregrinos jíbaros y su aprendiz. La experiencia es universalizable y no depende de ninguna magia. Harner, sin haber leído a Arguedas, apunta que "this is an experience of the non-ordinary as the sacred, not the sacred as o-ther, because this is a coming home" (14). En el caso de que este peregrinaje se hiciera incorrectamente, la experiencia podría resultar en muerte.

Se trata pues de una fuerza profunda y potente (14-19). La experiencia chamánica, que creo se da en la experiencia del afecto-cognitivo ante ciertos aspectos o eventos de la "naturaleza" en Arguedas y Hudson, trata de convergencias interespecie que permiten arribar a un hogar, a un lugar de gozo libre de amenazas y miedos. Estos encuentros nahualísticos y entradas en el mundo-espíritu, estos goces, se dan tanto en la vivencia del momento como en la memoria del momento que la escritura capta con acierto chamánico. No sabría decir qué parecido guarda este momento con la nómada del rizoma de Deleuze y Guattari.

\section{Conclusión}

Coincidiendo con la ambición de Deleuze y Guattari en A Thousand Plateaus de hacer de un texto un espacio rizomático en sí, se podría decir que la escritura material y espiritual de El zorro, o Los ríos profundos o Far Away and Long Ago es una especie de viaje chamánico. En Los ríos profundos aprendemos a apreciar esos espléndidos goces del encontrarse en casa y de simplemente sersiendo con un miembro de otra especie. Goces de saberse en una dimensión más cobijadora que cualquiera otra del mundo de la experiencia ordinaria. Se trata de una entrada al mundo-espíritu, a esa extensión del ser-siendo añorada por Arguedas. La escritura de El zorro podría tomarse a manera del oscuro descenso por un túnel, que cuando la experiencia es positiva nos lleva al encuentro saludable con el nahual, la restauración de la salud o el bienestar, pero que cuando falla nos inserta en un mundo de horror y sin salida. En Los ríos profundos, Ernesto, como Hudson, tiene o busca acceso a sus nahuales (power animals) y entrada al mundo-espíritu. Sus encuentros con sapos, loros, ríos, cascadas, ratas y flamencos son gloriosos. Para Arguedas, la dimensión de los nahuales incluye a los colonos, doña Felipa, los músicos de las chucherías y más tarde a las prostitutas de Chimbote y a los locos que predican en las calles del desierto.

Pero los danzantes sagrados del cuento "La agonía de Rasu Niti", en la zona envenenada de Chimbote, se han convertido en lugares de peligros, en seres que invitan a un peregrinaje en el cual se puede perder el peregrino, encontrarse en un túnel sin salida. Aunque es siempre posible que se preste el poder curativo de 
doña Felipa, de los músicos, al final, debe emprender su viaje y buscar su nahual, reencontrarse con su guardián -por medio del baile- para restablecer las fuerzas vitales positivas que la enfermad le ha mermado.

Cuando todas las entradas al mundo-espíritu fallan, tanto para Hudson como para Arguedas, queda la entrada por medio de la magia de la escritura, a la manera en que otros chamanes encuentran su poder en el viaje y el encuentro con su power animal, con quien deben bailar a menudo para no perder la conexión y mantener su poder. Esta transición del nahual al espacio de la escritura, si pudiera sustentarse, es problemática y difícil, y debo reflexionar más en ello. Lo que sí es claro en Arguedas y en algunos de sus personajes es que la música y la danza juegan la misma función de constituir la apertura sensorial-cognitiva por la cual el chamán entra y habita el estado de conciencia no-ordinario en que se le revelan conocimientos de una "cosmic geography of non-ordinary reality so that he may know where to journey to find the approriate animal, plant, and other powers" (Harner 21), que hacen posible que cure a sus pacientes y redoble su conexión con su animal-poder o guardián. En estos viajes el chamán ejercita todas las dimensiones del afecto-cognitivo, para así restaurar al paciente o a sí mismo la salud que las convergencias intraespecie permiten. El dansak, el escritor, el músico y el loco predicador hacen práctica de un modo de viaje chamánico restaurativo, y por lo tanto esencial a la vida. No veo un paralelismo entre la teorizada experiencia rizomática de Deleuze y Guattari y este viaje restaurativo abierto a cualquier miembro de la comunidad que tenga la voluntad o la suerte de encontrase con un guía conocedor de la peripecia empírica intraespecie. La experiencia chamánica, tal como se da en Arguedas y en Hudson, intenta restaurar o establecer la armonía y el equilibrio con la totalidad de la naturaleza, y creo que por lo tanto excede las dimensiones de conectividad imaginadas por Deleuze y Guattari.

\section{Obras citadas}

Arguedas, José María. Los ríos profundos. Buenos Aires: Losada, 1958.

Borges, Jorge Luis. Otras inquisiciones. Buenos Aires: Emecé, 1960.

- Obras completas, 1923-1972. Buenos Aires: Emecé, 1974.

Castro-Klarén, Sara. "Como chancho, cuando piensa'. El afecto cognitivo en Arguedas y el convertirse en animal". Revista Canadiense de Estudios Hispánicos XXXVI. 1-2. (2001-2002, otoño/invierno): 25-39.

Deleuze, Gilles y Felix Guattari. A Thousand Plateaus: Capitalism and Schizophrenia.

Trad. Brian Massumi. Minneapolis: University of Minnesota, 1987.

Gómez, Leila. Iluminados y tránsfugas. Relatos de viajeros y ficciones nacionales en Argentina, Paraguay y Perú. Madrid; Frankfurt: Iberoamericana; Vervuert, 2009. 
Harner, Michael. The Way of the Shaman: A Guide to Power and Healing. Nueva York: Harper and Row, 1979.

Hudson, William Henry. Far Away and Long Ago. A History of My Early Life. Nueva York: E.P. Dutton \& Co., 1931

Salomon, Frank, ed. The Huarochiri Manuscript: A Testament of Ancient and Colonial Andean Religion. Trad. George L. Urioste. Austin: University of Texas, 1991. Tylor, Edward. Primitive Culture: Researches into the Development of Mythology, Religion, Language, Arts and Custom. Nueva York: Brentano, 1871. 